\title{
Performance and Upgrades of the Fermilab Accumulator Stacktail Stochastic Cooling
}

\author{
P.F. Derwent, Ed Cullerton, David McGinnis, Ralph Pasquinelli, Ding Sun, \\ David Tinsley
}

Fermi National Accelerator Laboratory, P. O. Box 500, Batavia IL 60510-0500

\begin{abstract}
We report on the performance and planned upgrades to the Fermilab Accumulator Stacktail Stochastic Cooling System. The current system has achieved a maximum flux of 16.5e10/hour, limited by the input flux of antiprotons. The upgrades are designed to handle flux in excess of 40e10/hour.
\end{abstract}

Keywords: Stochastic Cooling, Antiproton Beams

PACS: 41.75.Lx

\section{THE FERMILAB ACCUMULATOR}

The Fermilab Accumulator is an $8 \mathrm{GeV}$ ring designed for the collection, cooling, and storage of anitprotons. The Tevatron Collider program requires 1e13 antiprotons for the study of proton-antiproton collisions at $\sqrt{s}=1.96 \mathrm{TeV}$. Antiprotons are produced by impinging a $120 \mathrm{GeV}$ proton beam on an nickel alloy target and collected through a lithium focussing lens and the Fermilab Debuncher ring [1]. As the number of antiprotons collected per pulse (which occur every 2 seconds) is on the order of a few $\times 10^{8}$, it is necessary to have a storage ring to collect and compress the phase space. For the last 20 years of the Tevatron Collider complex, the Fermilab Accumulator has served as that ring. With the advent of electron cooling in the Fermilab Recycler [2], the Accumulator will not be the final storage ring but will still play a significant role in the compression of the antiproton phase space.

\section{THEORY OF STOCHASTIC STACKING}

Stochastic stacking with a constant flux is achieved by designing a system with gain as a function of energy that falls exponentially, with characteristic energy $E_{d}$. The resulting density distribution then rises exponentially with the same characteristic energy [3]. The resulting maximum flux $\Phi$ can be expressed as:

$$
\Phi=\frac{W^{2}|\eta| E_{d}}{f_{0} p \ln \left(\frac{F_{\max }}{F_{\min }}\right)}
$$

where $W$ is the electronic bandwidth of the system, $\eta$ is the phase slip factor, $f_{0}$ is the beam revolution frequency, $p$ is the beam momentum, and $F_{\max }$ and $F_{\min }$ are the maximum and minimum frequencies in the system electronic bandwidth. Planar pickups 


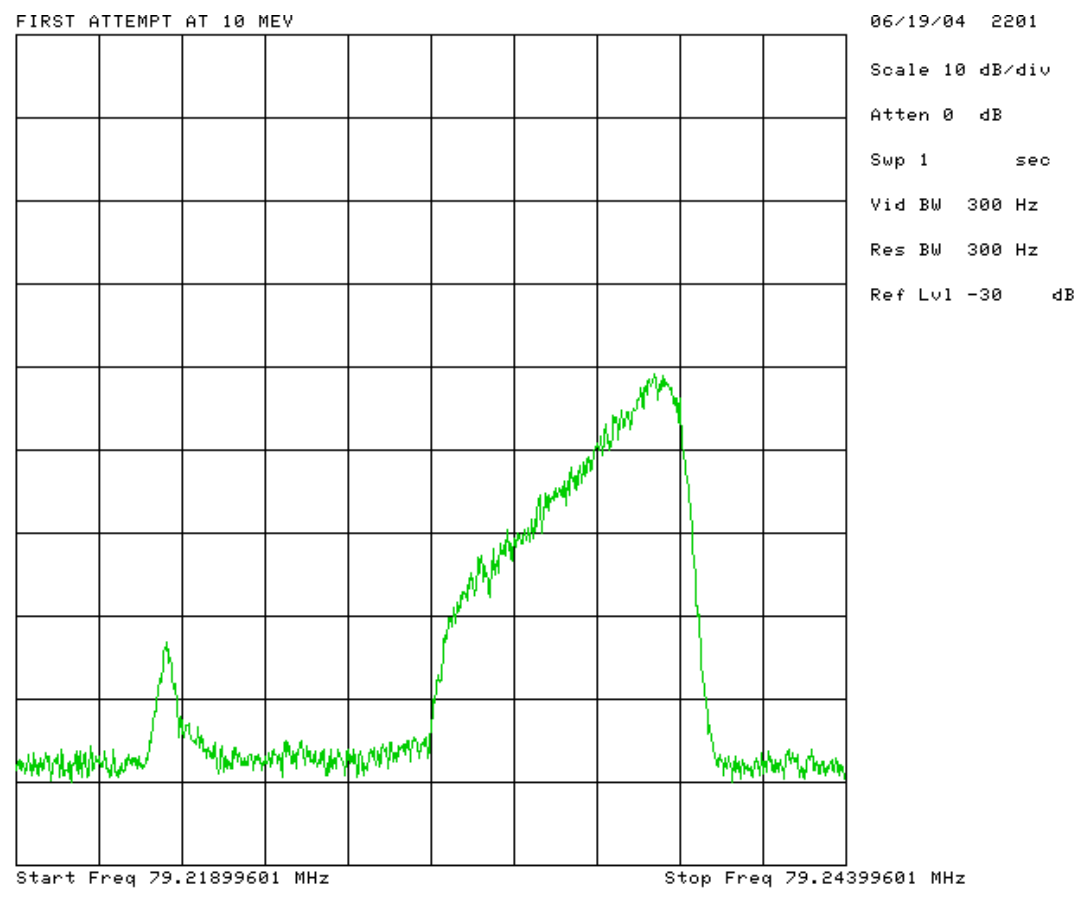

FIGURE 1. Current stacktail profile, which fits to a $E_{d}$ of $10 \mathrm{MeV}$.

have a response that follows $\exp \left(-\frac{\pi x}{d}\right)$ where $x$ is the transverse distance from the center of the pickup and $d$ is the vertical aperture. If the pickups are located in a region of high momentum dispersion, a system can be designed where the gain response falls off exponentially with energy. The region of exponential density increase is called the stacktail and the region where beam accumulates is called the core. As the revolution frequency changes with energy so does the flight time between pickup and kicker. The delay time through the electronics is a constant, hence, it is necessary to use multiple sets of pickups with different gains and delays to build the gain slope across the aperture. Correlator notch filters are used to null the signal at the core.

\section{CURRENT PERFORMANCE}

The present Accumulator provides a good example of the basic principles. Figure 1 shows the antiproton density distribution as a function of the beam revolution frequency, using a longitudinal Schottky pickup. We have 2 sets of pickups, located in a region with $10 \mathrm{~m}$ of dispersion, separated by $15 \mathrm{~mm}$ radially. The pickups and front end amplifiers are cooled by liquid Nitrogen, with an effective noise temperature of $125 \mathrm{~K}$. There are 256 pickup loops at $15 \mathrm{MeV}$ (with respect to the central energy of the Accumulator) and 48 pickup loops at $-8 \mathrm{MeV}$. There are 128 kicker loops in 8 tanks, with 4 TWTs per tank. There is approximately $150 \mathrm{~dB}$ of gain from pickup to kicker. By adjusting the relative gain and phase of the two sets of pickups, we have achieved an exponential gain slope of $10 \mathrm{MeV}$. The system bandwidth, accounting for phase variation as a function of frequency, is measured to be $1.2 \mathrm{GHz}$. With the machine parameters of the Accumulator, these values support a maximum flux of 29.5e10/hour. Integrating over an hour, our best performance has been the accumulation of $16.5 \mathrm{e} 10$. The system is still limited by the input flux. 


\section{UPGRADE DESIGN APPROACH}

Of the parameters appearing in Eq. ( 1$), E_{d}, W$, and $\eta$ are the only ones that can reasonably be considered as changeable. The simplest approach to maximize the flux is to increase $E_{d}$. This approach sacrifices the amount of density compaction achievable, since the density grows as $\exp \left(\frac{E}{E_{d}}\right)$, but has fewer implications for other systems in the Accumulator. Increasing the bandwidth clearly increases the maximum flux. Both approaches will be taken in this upgrade. Changes to $\eta$ are not being considered at this time.

A two-stage upgrade is planned to handle increased input flux [1]. In the initial stage, the characteristic energy $E_{d}$ is increased from $10 \mathrm{MeV}$ to $18 \mathrm{MeV}$. This change can be implemented with a minimal change in hardware through changes to pickup position (moving tanks radially in the Accumulator tunnel) and electronic gain and phase settings. The second stage requires additional pickups, electronics, and kickers; all covering the frequency range 4-6 GHz.

\section{DESIGN CONSTRAINTS}

There are drawbacks to increasing $E_{d}$. The Accumulator has a finite momentum aperture. It is therefore necessary to stop the flux at some point and accumulate it in a 'core'. The gain function will then deviate from a pure exponential and other considerations come to the fore. It is necessary to match the stacktail system gain to the core system gain to have a smooth transition in the gain profile. As the density increases for a given value of the gain, diffusive beam heating from other particles (through the cooling systems) eventually dominates the cooling term and the system no longer is able to effectively increase the density. It is generally true that as the density of the core increases it becomes necessary to decrease the system gain to maintain some margin between the cooling and diffusive terms in the Fokker-Planck equation.

Another limitation is the assumption of constant input flux. The input flux is a transient, with large pulses coming every 2 seconds. It is necessary for the input pulse to move completely into the stacktail region before the next pulse arrives or it will be phase displaced by the RF bucket moving the new pulse onto the deposition orbit. The fraction of the input pulse that moves across the aperture is a function of the gain of the system and the momentum distribution of the input pulse. The larger the gain, the more efficiently the input pulse moves off of the deposition orbit. The large gain necessary for effective stacking of the input pulse is also detrimental (for reasons given above) to accumulating large amounts of beam in the core.

\section{DESIGN CALCULATIONS}

Using a numerical integration of the Fokker-Planck equation, including models of pickup and kicker response, amplifier and notch filter performance, and a full implementation of the beam feedback terms [4], both stages of the upgrade have been simulated. The simulation designs for stage 1 (2-4 GHz bandwidth, $18 \mathrm{MeV}$ characteristic energy) 


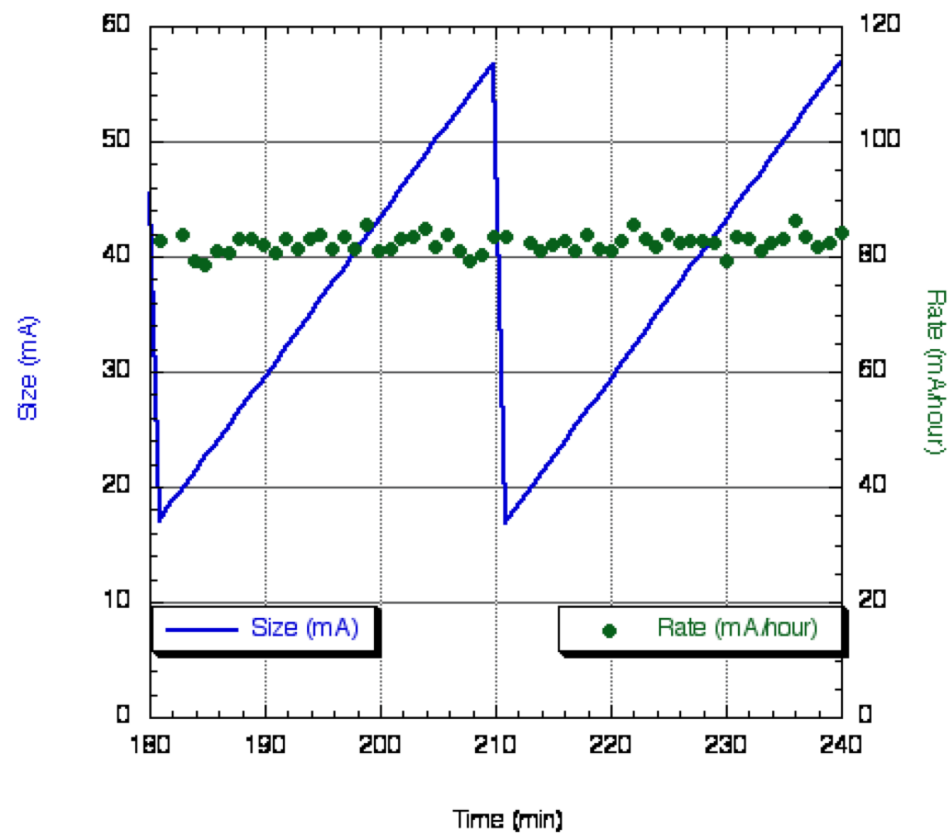

FIGURE 2. Stage 2 simulation results. The green dots are the accumulation rate per minute, the blue line is the beam current in the Accumulator. The input flux is approximately $80 \mathrm{e} 10 /$ hour, transfers to the Recycler occur every 30 minutes and take 1 minute to complete.

and stage 2 (2-6 GHz bandwidth, $10 \mathrm{MeV}$ characteristic energy) are complete. For stage 1 , the following changes are necessary:

- Move 256 pickups $1 \mathrm{~mm}$ radially outward from current location (an energy change of $1 \mathrm{MeV}$ )

- Move 48 pickups $7 \mathrm{~mm}$ radially outward from current location (an energy change of $8 \mathrm{MeV}$ )

- Adjust system gains and phases

With these changes, the stacktail can sustain a stack rate of $>55 \mathrm{e} 10 /$ hour for 30 minutes.

For stage 2, because of the increased bandwidth of the system, it is necessary to decrease the total energy change in the system to avoid Schottky band overlap. Hence, the core energy is closer to the stacktail. Half the $2-4 \mathrm{GHz}$ pickups and kickers are removed and replaced with 4-6 GHz pickups and kickers. With the increased bandwidth, the characteristic energy is lowered to approximately $15 \mathrm{MeV}$. The resulting system can sustain $80 \mathrm{e} 10 /$ hour for 30 minutes. The accumulation rate and beam current for this simulation are shown in figure 2.

\section{UPGRADE STATUS}

The current configuration of the stacktail is able to handle the current maximum input flux to the Accumulator. With the coming improvements [1], we anticipate that we will make use of the staged approach. The 4-6 GHz pickups and kickers have been designed and measured with a stretched wire, with the results agreeing well with microwave structure simulations. We are currently building a prototype tank to house several design iterations of the pickup loops. This tank will be installed in the Fermilab Debuncher this fall for beam tests. We anticipate assembly of the 4-6 GHz pickup and kicker tanks 
to take place during the 2006 calendar year, with installation during an appropriate accelerator shutdown period.

\section{CONCLUSIONS}

The Fermilab Accumulator stacktail design and performance has been described. The current arrangement can support a theoretical maximum flux of 29.5e10/hour, though the maximum value achieved has been limited by the input flux and is $16.5 \mathrm{e} 10$ in one hour. A two stage design approach has been presented, to handle increases of the input flux to 80e10/hour. Beam measurements are planned and we anticipate installation as the input flux outperforms the stacktail.

\section{REFERENCES}

1. D. McGinnis, "Antiproton production rate increase", these proceedings.

2. S. Nagaitsev, "Antiproton cooling in the Fermilab Recycler", these proceedings.

3. D. Möhl, et al., "Physics and Technique of Stochastic Cooling", Physics Reports, 58, No. 2 (1980).

4. J. Marriner \& V. Visnjic, "Fermilab Stochastic Cooling Code User's Guide", Fermilab Pbar Note 498 (unpublished), http : / / www-bdnew. fnal . gov/pbar/documents/pbarnotes / pdf_files/pb498.pdf. The Fortran code has been rewritten in $\mathrm{C}++$ for these simulations. 